Monatsschrift f. Geburtshülfe u. Gynäkologie 1923;64:I-IV

\title{
Contents, Vol. 64, 1923
}

\section{Inhaltsverzeichnis.}

S $\theta$ ite

Orïginalarbeiten.

Borchardt, L.. Über thyreosexuelle Insuffizienz . . . 253 Dyroff, R., Zur Vagitusfrage und zur Frage der Asphyxie 75 Falk, R., Intra- oder extraovuläre Metreuryse bei Placenta praevia 145

Fekete, A. v., Die Störungen der Menstruation und der

Einfluß von Organextrakten auf dieselben 267

Fink, Karl, Über Entstehung und Bedeutung des Ligamentum rectovesicale 263

Fink, K., Behandlung des Puerperalfiebers 305

Franz, Th., Über Plazentarstoffe und die motorische Funk-

tion des Uterus

7

Frigyesi, J. u. A. Lang, L·ber Versuche mit der Tiefen-

desinfektion der Gewebe bei der Behandlung und

Operation septischer Erkrankungen der weiblichen

Genitalorgane 85

Gamper, A., Zangenfrequenz und Kindersterblichkeit. . 297 Grabieh, F., Aktive oder abwartende Behandlung der

septischen Aborte 308

Gragert, 0., Wochenbettsniorbidität bei ante partum.

nichtbehandelten und ante partum behandelten Fallen

von Trichomonaden-Kolpitis 37

Greil, A., Entstehung angeborener Knochenbrüchigkeit . 59 Guthmann, H. , Weitere

Erfahrungen mit der Eileiter-

durchblasung (Pertubation) 55

Haupt, W., Zur Behandlung der Pyelitis

Keitler, H., Über einen Fall von Nabeladenom. Mit Be-

merkung en über vikarierende Menstruation 171

Kermauner, F., Dehnungsstreifen der Haut 125

Kraul, L., Zur Vakzinetherapie der Gonorrhöe beim Weibe 203 Küstner, H., Isolierte Metastase eines primären Ovarial-

karzinoms in der Zervix und Portio uteri

193

Lederer, L.. Über die Beziehimgen der Tuboo $\lambda^{3} / 4$ rialzysten

zur Salpingitis isthmica nodosa

45

Lindemann, W., Über rationelle Anwendung der gynäko-logischen Diathermie und ihre spezielle Verwendung

bei Zervixgonorrhöe 333

Liss, $\Lambda$ V., Der Einfluß der Trichomonadenkolpitis auf die 
Wochenbettsniorbidität

31

Matsuno, Joshimitsu, Zur Kenntnis der Funktion des Corpus luteum. (Zugleich ein Beitrag zur Autoimplanta-

tion des prolabierten Corpus luteum.) -

Mayer, A., Über die biologische Einheit zwischen Mutter

und Kind 131

-. Über angeborenen Ikterus 135

*

$\mathrm{J}[\gamma \quad$ Inhaltsverzeichnis.

Seite

$0 \mathrm{p} p$ e $\mathrm{n}$ h e im e r, W., Zur Frage der Bedeutung des Ikterus

bei Eklampsie 289

Richter, J., Besitzt das weibliche Genitale speicherungs-

fähige Retikuloendothelien ? Ein Beitrag zur Frage der

Therapie des Puerperalfiebers 323

Schmidt, H. R., Zur Bewertung der Herzfehler in der

Schwangerschaft 279

Schneider, G. H., Schwangerschaft nach doppelseitiger

Mammaamputation 295

-, Rezidivierende Schwangerschaftsakne 42

Sellheim, H. , Zum leichteren Verständnis des hydrau-

lischen Druckes unter der Geburt 1

Stein, F., Die aktive Behandlung fieberhafter Aborte in

ihrem Einfluß auf Fieber-, Blutungs- und Behandlungs-

dauer 155

Strube, H., Prophylaxe der ChloroEormsynkope . . . 351 Unterberger, F.,

Keimdrüsenbestrahlung und Nach-

kommenschaft 211

Weinsheimer, K., Erfahrungen mit der wirksamen

Sekalesubstanz $\quad 149$

Sammelberichíe.

Die gynäkologische Strahlentherapie im Jahre 1922. Kri-

tischer Bericht von H. hanger in Erlangen . . . . 217

Goethe über Anfang, Entwicklung und Ende der Schwanger-

$\mathrm{i}^{3} / 8 \mathrm{chaft}$

243

Paul Mathes $\uparrow 243$

Vereins- und Líteraturbeilage.

48. Kongreß der American Gynecological Society. Original-

bericht von Prof. Dr. 0. Franl·1 in Wien 93

Vereinigung zur Fòrderung des deutschen Hebammenwesens 96 Nachtrag zum Bericht von

Eufinger über die Heidelberger

Verhandlungen 242

Original-Sitzungsberichte aus geburtshớlflich-gynäkologischen Gesellschafte $\pi$ : Gesellschaft für Geburtshülfe und Gynäkologie in Berlin.

Sitzg. v. 16. Febr., 9., 23. März, 8. Juni u. 22. Juni 1923 101, 365 Nordostdeutsche Gesellschaft für Gynäkologie in Königs- 
berg i. Pr. Sitzung vom 14. April 1923

105

-, Sitzung vom 23. Juni 1923355

Geburtshülflich-gynäkologische Gesellschaft in Wien. Sitzung

vom 13. Februar «nd 13. März 1923360

Münchener Gynäkologische Gesellschaft. Sitzung v. 22. Febr.

$1923 \quad 361$

Reîerate aus geburtshülflích-gynäkolog. Gesellschafte $\pi$ 119, 245, 371 Geburtshülflichgynäkologische Mitteilungen aus anderen me-

diziníschen Gesellschaften und Vereinen 121, 372

Líteratur-Verzeíchnís 122, 249, 372

Personalien 376

Berichtigung 376 